\title{
GENERAL TAX STRUCTURES AND THE LÉVY INSURANCE RISK MODEL
}

\author{
ANDREAS E. KYPRIANOU, ${ }^{*}$ The University of Bath \\ XIAOWEN ZHOU, ${ }^{* *}$ Concordia University
}

\begin{abstract}
In the spirit of Albrecher and Hipp (2007), and Albrecher, Renaud, and Zhou (2008) we consider a Lévy insurance risk model with tax payments of a more general structure than in the aforementioned papers, which was also considered in Albrecher, Borst, Boxma, and Resing (2009). In terms of scale functions, we establish three fundamental identities of interest which have stimulated a large volume of actuarial research in recent years. That is to say, the two-sided exit problem, the net present value of tax paid until ruin, as well as a generalized version of the Gerber-Shiu function. The method we appeal to differs from Albrecher and Hipp (2007), and Albrecher, Renaud, and Zhou (2008) in that we appeal predominantly to excursion theory.
\end{abstract}

Keywords: Reflected Lévy process; passage problem; integrated exponential Lévy process; insurance risk process; ruin; excursion theory

2000 Mathematics Subject Classification: Primary 60K05; 60K15; 91B30

Secondary 60G70; 60J55

\section{Introduction and main results}

Recent advances in the analysis of the ubiquitous ruin problem from the theory of insurance risk has seen a tendency to replace the classical Cramér-Lundberg surplus process with a general spectrally negative Lévy process; see, for example, [2], [10], [11], and [21], to name but a few. In this case the surplus process is commonly referred to as a Lévy insurance risk process. Although moving to this more complex setting, arguably, does not bring any more realistic features to the table than are already on offer in the classical Cramér-Lundberg model, a clear mathematical advantage has emerged. Working with Lévy insurance risk processes forces us to approach the problem of ruin via fluctuation theory, which does not use specific features of the underlying Lévy process other than a generic path decomposition of the process in terms of excursions from its maximum, which manifests itself in the form of a Poisson point process.

In this paper we continue in this vein and build on ideas of Lévy insurance risk processes with tax which were introduced and studied in [1], [2], and [3]. Specifically, we introduce a more general tax structure and therewith we establish, for the aggregate surplus process, new identities for the two-sided exit problem, a generalized version of the Gerber-Shiu function, as well as the net present value of tax paid until ruin.

Received 3 February 2009; revision received 22 July 2009.

* Postal address: Department of Mathematical Sciences, The University of Bath, Claverton Down, Bath BA2 7AY, UK. Email address: a.kyprianou@bath.ac.uk

** Postal address: Department of Mathematics and Statistics, Concordia University, 1455 De Maisonneuve Blvd. West, Montreal Quebec, H3G 1M8, Canada. Email address: xzhou@ mathstat.concordia.ca 
Henceforth, the process $X=\left\{X_{t}: t \geq 0\right\}$ with probabilities $\left\{\mathrm{P}_{x}: x \in \mathbb{R}\right\}$ and natural filtration $\left\{\mathcal{F}_{t}: t \geq 0\right\}$ will denote a spectrally negative Lévy process with the usual exclusion of processes in the latter class which have monotone paths (that is to say, a pure increasing linear drift and the negative of a subordinator). For convenience, we shall always denote $\mathrm{P}_{0}$ by $\mathrm{P}$. Let

$$
\psi(\theta)=\log \mathrm{E}\left[\mathrm{e}^{\theta X_{1}}\right]
$$

be the Laplace exponent of $X$, which is known to be finite for at least $\theta \in[0, \infty)$, in which case it is a strictly convex and infinitely differentiable function. The asymptotic behaviour of $X$ is characterized by $\psi^{\prime}(0+)$, so that $X$ drifts to $\pm \infty$ or oscillates according to whether $\pm \psi^{\prime}(0+)>0$ or, respectively, $\psi^{\prime}(0+)=0$. When $X$ plays the role of the surplus process, it is usual to assume that $\psi^{\prime}(0+)>0$, which is equivalent to the net profit condition in the case that $X$ is a Cramér-Lundberg process. However, this condition is not necessary for any of the forthcoming analysis.

Denote by $S=\left\{S_{t}: t \geq 0\right\}$ the process which describes the running supremum of $X$, that is to say, $S_{t}=\sup _{s \leq t} X_{s}$ for each $t \geq 0$. Following [3], we are interested in modelling tax payments from the Lévy insurance process in such a way that the cumulative payment until time $t$ is given by

$$
\int_{0}^{t} \gamma\left(S_{u}\right) \mathrm{d} S_{u}
$$

where $\gamma:[0, \infty) \rightarrow[0,1)$ is a measurable function which satisfies

$$
\int_{0}^{\infty}(1-\gamma(s)) \mathrm{d} s=\infty .
$$

In this case the aggregate surplus process, the primary object of our study, is given by

$$
U_{t}:=X_{t}-\int_{0}^{t} \gamma\left(S_{u}\right) \mathrm{d} S_{u} .
$$

In the special case that $\gamma$ is a constant in $(0,1)$, our Lévy insurance risk process with tax agrees with the model introduced in [1] and [2]. In the case that $\gamma=0$, we are back to a regular Lévy insurance risk process. Note also that processes of the form (1.1) constitute a subclass of controlled Lévy risk processes, the latter being of popular interest in recent literature; see, for example, [5], [13], and [14].

In order to state the results alluded to above which concern path functionals of $U$, we must first introduce more notation. As is now usual when studying Lévy risk processes, a key element of the analysis involves the use of scale functions, defined as follows. For every $q \geq 0$, there exists a function $W^{(q)}: \mathbb{R} \rightarrow[0, \infty)$ such that $W^{(q)}(x)=0$ for all $x<0$ and otherwise is almost everywhere differentiable on $[0, \infty)$, satisfying

$$
\int_{0}^{\infty} \mathrm{e}^{-\lambda x} W^{(q)}(x) \mathrm{d} x=\frac{1}{\psi(\lambda)-q} \text { for } \lambda>\Phi(q),
$$

where $\Phi(q)$ is the largest solution to the equation $\psi(\theta)=q$ (there are at most two). We shall write for short $W^{(0)}=W$. It is known that when $X$ has paths of unbounded variation, the scale functions $W^{(q)}$ are continuously differentiable on $(0, \infty)$, and when $X$ has paths of bounded variation, they are almost everywhere differentiable. In either case we shall denote by $W^{(q)^{\prime}}$ the associated density. It is also known that if $X$ has a Gaussian component then $W^{(q)}$ is 
twice continuously differentiable on $(0, \infty)$. In the case of bounded variation paths, there is a discontinuity in $W^{(q)}$ at the origin which does not depend on $q$, and in the case of unbounded variation paths, there is no discontinuity in $W^{(q)}$ at the origin for all $q \geq 0$.

There exists a well-known exponential change of measure that we may perform for spectrally negative Lévy processes,

$$
\left.\frac{\mathrm{dP}_{x}^{\vartheta}}{\mathrm{dP}_{x}}\right|_{\mathcal{F}_{t}}=\mathrm{e}^{\vartheta\left(X_{t}-x\right)-\psi(\vartheta) t}
$$

for $x \in \mathbb{R}$ and $\vartheta \geq 0$, under which $X$ remains within the class of spectrally negative Lévy processes. In particular, if $v(\mathrm{~d} x)$ is the Lévy measure of $-X$ under $\mathrm{P}$ then $\mathrm{e}^{-\vartheta x} v(\mathrm{~d} x)$ is its Lévy measure under $\mathrm{P}^{\vartheta}$. It will turn out to be useful to introduce an additional parameter to the scale functions described above in the light of this change of measure. Henceforth, we shall refer to the functions $W_{\vartheta}$, where $\vartheta \geq 0$, as the functions that play the role of the scale functions defined in the previous paragraph, but when considered under the measures $\mathrm{P}^{\vartheta}$.

Next define

$$
\tau_{a}^{+}:=\inf \left\{t>0: U_{t}>a\right\} \quad \text { and } \quad \tau_{0}^{-}:=\inf \left\{t>0: U_{t}<0\right\}
$$

with the convention inf $\varnothing=\infty$. For $s \geq x$, define

$$
\bar{\gamma}(s):=s-\int_{x}^{s} \gamma(y) \mathrm{d} y=x+\int_{x}^{s}(1-\gamma(y)) \mathrm{d} y .
$$

By differentiating (1.4) we note that, since $\gamma \in[0,1)$, it follows that $\bar{\gamma}$ is a strictly increasing function. Moreover, since it is continuous, it has a well-defined inverse on $[x, \infty)$ which we denote by $\bar{\gamma}^{-1}$.

We may now present the three main results of this paper. Their proofs will be given in the subsequent sections.

For each $x>0$, the process $L_{t}:=S_{t}-x, t \geq 0$, serves as a local time at 0 for the Markov process $Y:=S-X$ under $\mathrm{P}_{x}$. Write $L^{-1}:=\left\{L_{t}^{-1}: t \geq 0\right\}$ for the right-continuous inverse of $L$.

Theorem 1.1. (Two-sided exit problem.) For any $x>0$ and $q \geq 0$, we have

$$
\mathrm{E}_{x}\left[\mathrm{e}^{-q \tau_{a}^{+}} \mathbf{1}_{\left\{\tau_{a}^{+}<\tau_{0}^{-}\right\}}\right]=\exp \left\{-\int_{x}^{a \wedge x} \frac{W^{(q) \prime}(y)}{W^{(q)}(y)\left(1-\gamma\left(\bar{\gamma}^{-1}(y)\right)\right)} \mathrm{d} y\right\} .
$$

Theorem 1.2. (Net present value of tax paid until ruin.) For any $x>0$ and $q \geq 0$, we have

$$
\mathrm{E}_{x}\left[\int_{0}^{\tau_{0}^{-}} \mathrm{e}^{-q u} \gamma\left(S_{u}\right) \mathrm{d} S_{u}\right]=\int_{x}^{\infty} \exp \left\{-\int_{x}^{t} \frac{W^{(q) \prime}(\bar{\gamma}(s))}{W^{(q)}(\bar{\gamma}(s))} \mathrm{d} s\right\} \gamma(t) \mathrm{d} t .
$$

Theorem 1.3. For each $t \geq 0$, let $S_{t}^{U}:=\sup _{s \leq t} U_{s}$ and let $\kappa=L_{\tau_{\tau^{-}}^{-}}^{-1}$, the last moment that tax is paid before ruin. Denote by $v$ the Lévy measure of $-X$. For any $z>0, y \geq 0, \theta \geq y$, 
and $\alpha, \beta \geq 0$, we have

$$
\begin{aligned}
\mathrm{E}_{x}\left(\mathrm{e}^{-\alpha \kappa-\beta\left(\tau_{0}^{-}-\kappa\right)} ; S_{\tau_{0}^{-}}^{U} \in \mathrm{d} \theta, U_{\tau_{0}^{-}-} \in \mathrm{d} y,-U_{\tau_{0}^{-}} \in \mathrm{d} z\right) \\
=\frac{1}{1-\gamma\left(\bar{\gamma}^{-1}(\theta)\right)} \exp \left\{-\int_{x}^{\theta} \frac{W^{(\alpha) \prime}(v)}{W^{(\alpha)}(v)\left(1-\gamma\left(\bar{\gamma}^{-1}(v)\right)\right)} \mathrm{d} v\right\} \\
\times\left[\left\{W^{(\beta)^{\prime}}(\theta-y)-\frac{W^{(\beta) \prime}(\theta)}{W^{(\beta)}(\theta)} W^{(\beta)}(\theta-y)\right\} v(y+\mathrm{d} z) \mathbf{1}_{\{y<\theta\}} \mathrm{d} y\right. \\
\left.\quad+W^{(\beta)}(0+) v(\theta+\mathrm{d} z) \delta_{\theta}(\mathrm{d} y)\right] \mathrm{d} \theta,
\end{aligned}
$$

where $\delta_{\theta}(\mathrm{d} y)$ is the Dirac measure which assigns unit mass to the point $\theta$. Furthermore, we also have

$$
\begin{aligned}
\mathrm{E}_{x}\left(\mathrm{e}^{-\alpha \kappa-\beta\left(\tau_{0}^{-}-\kappa\right)} ; S_{\tau_{0}^{-}}^{U} \in \mathrm{d} \theta, U_{\tau_{0}^{-}}=0\right) \\
=\frac{1}{1-\gamma\left(\bar{\gamma}^{-1}(\theta)\right)} \exp \left\{-\int_{x}^{\theta} \frac{W^{(\alpha) \prime}(y)}{W^{(\alpha)}(y)\left(1-\gamma\left(\bar{\gamma}^{-1}(y)\right)\right)} \mathrm{d} y\right\} \\
\quad \times \frac{\sigma^{2}}{2}\left\{\frac{W^{(\beta) \prime}(\theta)^{2}}{W^{(\beta)}(\theta)}-W^{(\beta) \prime \prime}(\theta)\right\} \mathrm{d} \theta,
\end{aligned}
$$

where $\sigma$ is the Gaussian coefficient in the Lévy-Itô decomposition of X.

Remark 1.1. When $\gamma \in(0,1)$ is a constant, we note that expressions (1.5) and (1.6) agree with formulae (3.1) and (3.2) of [2]. Indeed, we have $\bar{\gamma}(s)=s(1-\gamma)+\gamma x$. For Theorem 1.1, we have

$$
\mathrm{E}_{x}\left[\mathrm{e}^{-q \tau_{a}^{+}} \mathbf{1}_{\left\{\tau_{a}^{+}<\tau_{0}^{-}\right\}}\right]=\exp \left\{-\int_{x}^{a} \frac{W^{(q) \prime}(y)}{W^{(q)}(y)(1-\gamma)} \mathrm{d} y\right\}=\left(\frac{W^{(q)}(x)}{W^{(q)}(a)}\right)^{1 /(1-\gamma)} .
$$

For Theorem 1.2, we have, by two changes of variables,

$$
\begin{aligned}
\int_{x}^{\infty} \exp \left\{-\int_{x}^{t} \frac{W^{(q) \prime}(\bar{\gamma}(s))}{W^{(q)}(\bar{\gamma}(s))} \mathrm{d} s\right\} \gamma(t) \mathrm{d} t & =\gamma \int_{x}^{\infty} \exp \left\{-\frac{1}{1-\gamma} \int_{x}^{\bar{\gamma}(t)} \frac{W^{(q) \prime}(y)}{W^{(q)}(y)} \mathrm{d} y\right\} \mathrm{d} t \\
& =\gamma \int_{x}^{\infty}\left(\frac{W^{(q)}(x)}{W^{(q)}(\bar{\gamma}(t))}\right)^{1 /(1-\gamma)} \mathrm{d} t \\
& =\frac{\gamma}{1-\gamma} \int_{x}^{\infty}\left(\frac{W^{(q)}(x)}{W^{(q)}(u)}\right)^{1 /(1-\gamma)} \mathrm{d} u,
\end{aligned}
$$

which is Equation (3.2) of [2].

Theorem 1.3 on the other hand gives a new result for the setting of [2]. In particular, we have, for $y, z>0$ and $y \leq \theta$,

$$
\begin{aligned}
\mathrm{E}_{x}\left(\mathrm{e}^{-\alpha \kappa-\beta\left(\tau_{0}^{-}-\kappa\right)} ; S_{\tau_{0}^{-}}^{U} \in \mathrm{d} \theta, U_{\tau_{0}^{-}-}\right. & \left.\in \mathrm{d} y,-U_{\tau_{0}^{-}} \in \mathrm{d} z\right) \\
=\frac{1}{1-\gamma}\left(\frac{W^{(\alpha)}(x)}{W^{(\alpha)}(\theta)}\right)^{1 /(1-\gamma)}[ & \left\{W^{(\beta)^{\prime}}(\theta-y)-\frac{W^{(\beta) \prime}(\theta)}{W^{(\beta)}(\theta)} W^{(\beta)}(\theta-y)\right\} v(y+\mathrm{d} z) \\
& \left.\times \mathbf{1}_{\{y<\theta\}} \mathrm{d} y+W^{(\beta)}(0+) v(\theta+\mathrm{d} z) \delta_{\theta}(\mathrm{d} y)\right] \mathrm{d} \theta
\end{aligned}
$$


and

$$
\begin{aligned}
& \mathrm{E}\left(\mathrm{e}^{-\alpha \kappa-\beta\left(\tau_{0}^{-}-\kappa\right)} ; S_{\tau_{0}^{-}}^{U} \in \mathrm{d} \theta, U_{\tau_{0}^{-}}=0\right) \\
& \quad=\frac{\sigma^{2}}{2(1-\gamma)}\left(\frac{W^{(\alpha)}(x)}{W^{(\alpha)}(\theta)}\right)^{1 /(1-\gamma)}\left\{\frac{W^{(\beta) \prime}(\theta)^{2}}{W^{(\beta)}(\theta)}-W^{(\beta) \prime \prime}(\theta)\right\} \mathrm{d} \theta .
\end{aligned}
$$

Finally, note that, when $\gamma=0$ and the process $U$ agrees with the Lévy insurance risk process $X$, the last two formulae above give us two new expressions for the time value of the overall maximal wealth accumulated prior to ruin, the wealth immediately before ruin, and the deficit at ruin.

Remark 1.2. Following a preprint of this article, further identities concerning the process $U$ have been treated by Renaud [20] using a different technique.

Remark 1.3. One major criticism of working with scale functions is that, in principle, we have only solved the problems of interest up to inverting the Laplace transform (1.2). However, in the last year there have been a number of developments in the theory of scale functions which has seen a large number of explicit examples appearing in the literature, including the case of Cramér-Lundberg models. See, for example, [8], [9], [15], and [17]. Surya [22] gave recipes for evaluating scale functions numerically.

\section{Proofs of main results}

We begin this section by pointing out some important features of the running supremum of the aggregate process (1.1) which turns out to be key in our use of excursion theory in the forthcoming proofs.

Lemma 2.1. We have

$$
S_{t}^{U}=S_{t}-\int_{0}^{t} \gamma\left(S_{S}\right) \mathrm{d} S_{s}
$$

and the random times $\left\{t \geq 0: U_{t}=S_{t}^{U}\right\}$ agree precisely with $\left\{t \geq 0: X_{t}=S_{t}\right\}$.

Proof. Note that, on the one hand,

$$
S_{t}^{U}=\sup _{s \leq t}\left\{X_{s}-\int_{0}^{s} \gamma\left(S_{u}\right) \mathrm{d} S_{u}\right\} \geq \sup _{s \leq t} X_{s}-\int_{0}^{t} \gamma\left(S_{u}\right) \mathrm{d} S_{u}=S_{t}-\int_{0}^{t} \gamma\left(S_{u}\right) \mathrm{d} S_{u} .
$$

On the other hand, since $X_{t} \leq S_{t}$, we have

$$
U_{t} \leq S_{t}-\int_{0}^{t} \gamma\left(S_{u}\right) \mathrm{d} S_{u}=\int_{0}^{t}\left(1-\gamma\left(S_{u}\right)\right) \mathrm{d} S_{u}+x
$$

and, hence, since $\gamma(y) \in[0,1)$ for all $y \geq 0$,

$$
S_{t}^{U} \leq \sup _{s \leq t} \int_{0}^{s}\left(1-\gamma\left(S_{u}\right)\right) \mathrm{d} S_{u}+x=\int_{0}^{t}\left(1-\gamma\left(S_{u}\right)\right) \mathrm{d} S_{u}+x=S_{t}-\int_{0}^{t} \gamma\left(S_{u}\right) \mathrm{d} S_{u} .
$$

Together, (2.2) and (2.3) imply (2.1). Now suppose that $t^{\prime} \in\left\{t \geq 0: X_{t}=S_{t}\right\}$. This implies that

$$
U_{t^{\prime}}=X_{t^{\prime}}-\int_{0}^{t^{\prime}} \gamma\left(S_{u}\right) \mathrm{d} S_{u}=S_{t^{\prime}}-\int_{0}^{t^{\prime}} \gamma\left(S_{u}\right) \mathrm{d} S_{u}=S_{t^{\prime}}^{U}
$$


and, hence, $t^{\prime} \in\left\{t \geq 0: U_{t}=S_{t}^{U}\right\}$. On the other hand, if $t^{\prime \prime} \in\left\{t \geq 0: U_{t}=S_{t}^{U}\right\}$ then

$$
X_{t^{\prime \prime}}-\int_{0}^{t^{\prime \prime}} \gamma\left(S_{u}\right) \mathrm{d} S_{u}=U_{t^{\prime \prime}}=S_{t^{\prime \prime}}^{U}=S_{t^{\prime \prime}}-\int_{0}^{t^{\prime \prime}} \gamma\left(S_{u}\right) \mathrm{d} S_{u},
$$

showing that $X_{t^{\prime \prime}}=S_{t^{\prime \prime}}$ and, hence, $t^{\prime \prime} \in\left\{t \geq 0: X_{t}=S_{t}\right\}$.

For the remaining proofs, we shall also make heavy use of excursion theory for the process $S-X$; we refer the reader to [6, Chapters 6 and 7] for background reading. We shall spend a moment here setting up some necessary notation which will be used throughout the remainder of the paper. The Poisson point process of excursions indexed by local time shall be denoted by $\left\{\left(t, \varepsilon_{t}\right): t \geq 0\right\}$, where

$$
\varepsilon_{t}=\left\{\varepsilon_{t}(s):=X_{L_{t}^{-1}}-X_{L_{t-}^{-1}+s}: 0<s \leq L_{t}^{-1}-L_{t-}^{-1}\right\}
$$

whenever $\sigma\left(\varepsilon_{t}\right):=L_{t}^{-1}-L_{t-}^{-1}>0$. Accordingly, we refer to a generic excursion as $\varepsilon(\cdot)$ (or just $\varepsilon$ for short as appropriate) belonging to the space $\varepsilon$ of canonical excursions. The intensity measure of the process $\left\{\left(t, \varepsilon_{t}\right): t \geq 0\right\}$ is given by $\mathrm{d} t \times \mathrm{d} n$, where $n$ is a measure on the space of excursions (the excursion measure). An $n$-measurable functional of the canonical excursion which will be of prime interest is $\bar{\varepsilon}=\sup _{s \geq 0} \varepsilon(s)$. A useful formula for this functional that we shall make use of is the following (cf. [12, Equation (8.18)]):

$$
n(\bar{\varepsilon}>x)=\frac{W^{\prime}(x)}{W(x)}
$$

providing that $x$ is not a point of discontinuity in the derivative of $W$ (which is only a concern when $X$ has paths of bounded variation, in which case there are at most a countable number).

Lemma 2.1 also has an important bearing on the process of excursions described above. Indeed, from identity (2.1) we note that $L_{t}=s$, or, equivalently, $S_{t}=x+s$, under $\mathrm{P}_{x}$ if and only if $S_{t}^{U}=\bar{\gamma}(x+s)$. It follows that the event that $U$ climbs from $x$ to height $\bar{\gamma}(x+s)$ for the first time coincides with the event that process $X$ climbs from $x$ to $x+s$ for the first time. Consequently, $L_{s}^{-1}=\tau_{\bar{\gamma}(x+s)}^{+}$, or, equivalently, $\tau_{a}^{+}=L_{\bar{\gamma}^{-1}(a)-x}^{-1}$, under $\mathrm{P}_{x}$. Moreover, the excursions of $U$ away from its maximum agree precisely with $\left\{\left(t, \varepsilon_{t}\right): t \geq 0\right\}$.

Proof of Theorem 1.1. Taking account of the remarks following Lemma 2.1 we find that the event $\left\{\tau_{a}^{+}<\tau_{0}^{-}\right\}$is the same as

$$
\left\{\bar{\varepsilon}_{s} \leq \bar{\gamma}(x+s) \text { for all } 0 \leq s<\bar{\gamma}^{-1}(a)-x\right\}
$$

Then, for $x>0$,

$$
\begin{aligned}
\mathrm{P}_{x}\left(\tau_{a}^{+}<\tau_{0}^{-}\right) & =\mathrm{P}_{x}\left(\bar{\varepsilon}_{s} \leq \bar{\gamma}(x+s) \text { for all } 0 \leq s<\bar{\gamma}^{-1}(a)-x\right) \\
& =\exp \left\{-\int_{0}^{\bar{\gamma}^{-1}(a)-x} n(\bar{\varepsilon}>\bar{\gamma}(x+s)) \mathrm{d} s\right\} \\
& =\exp \left\{-\int_{0}^{\bar{\gamma}^{-1}(a)-x} \frac{W^{\prime}(\bar{\gamma}(x+s))}{W(\bar{\gamma}(x+s))} \mathrm{d} s\right\} \\
& =\exp \left\{-\int_{x}^{a} \frac{W^{\prime}(y)}{W(y)\left(1-\gamma\left(\bar{\gamma}^{-1}(y)\right)\right)} \mathrm{d} y\right\},
\end{aligned}
$$


where in changing variable we make use of the fact that, since $\bar{\gamma}\left(\bar{\gamma}^{-1}(s)\right)=s$, we have, from the chain rule,

$$
\frac{\mathrm{d}}{\mathrm{d} s} \bar{\gamma}^{-1}(s)=\frac{1}{\bar{\gamma}^{\prime}\left(\bar{\gamma}^{-1}(s)\right)}=\frac{1}{1-\gamma\left(\bar{\gamma}^{-1}(s)\right)} .
$$

Next, note that

$$
\begin{aligned}
\mathrm{P}_{x}^{\Phi(q)}\left(\tau_{a}^{+}<\tau_{0}^{-}\right) & =\mathrm{E}_{x}\left[\exp \left\{\Phi(q)\left(X_{\tau_{a}^{+}}-x\right)-q \tau_{a}^{+}\right\} \mathbf{1}_{\left\{\tau_{a}^{+}<\tau_{0}^{-}\right\}}\right] \\
& =\exp \left\{\Phi(q)\left(\bar{\gamma}^{-1}(a)-x\right)\right\} \mathrm{E}_{x}\left[\mathrm{e}^{-q \tau_{a}^{+}} \mathbf{1}_{\left\{\tau_{a}^{+}<\tau_{0}^{-}\right\}}\right]
\end{aligned}
$$

where we have appealed to the change of measure (1.3) with $\vartheta=\Phi(q)$ and the final equality follows by virtue of the fact that, on $\left\{\tau_{a}^{+}<\infty\right\}$,

$$
\begin{aligned}
X_{\tau_{a}^{+}} & =U_{\tau_{a}^{+}}+\int_{0}^{\tau_{a}^{+}} \gamma\left(S_{u}\right) \mathrm{d} S_{u} \\
& =a+\int_{0}^{L_{\bar{\gamma}}^{-1}(a)-x} \gamma\left(S_{u}\right) \mathrm{d} S_{u} \\
& =a+\int_{x}^{\bar{\gamma}^{-1}(a)} \gamma(y) \mathrm{d} y \\
& =\bar{\gamma}^{-1}(a),
\end{aligned}
$$

where in the third equality we have made the change of variable $y=S_{u}^{-1}$. Note also that it is known (cf. Chapter 8 of [12]) that, for $q, x \geq 0$,

$$
W^{(q)}(x)=\mathrm{e}^{\Phi(q) x} W_{\Phi(q)}(x)
$$

and, hence,

$$
\frac{W_{\Phi(q)}^{\prime}(x)}{W_{\Phi(q)}(x)}=\frac{W^{(q)^{\prime}}(x)}{W^{(q)}(x)}-\Phi(q) .
$$

Piecing together (2.5), (2.6), and (2.7) we obtain

$$
\begin{aligned}
& \mathrm{E}_{x}\left[\mathrm{e}^{-q \tau_{a}^{+}} \mathbf{1}_{\left\{\tau_{a}^{+}<\tau_{0}^{-}\right\}}\right] \\
& \quad=\mathrm{P}_{x}^{\Phi(q)}\left(\tau_{a}^{+}<\tau_{0}^{-}\right) \exp \left\{-\Phi(q)\left(\bar{\gamma}^{-1}(a)-x\right)\right\} \\
& \quad=\exp \left\{-\int_{x}^{a} \frac{W_{\Phi(q)}^{\prime}(y)}{W_{\Phi(q)}(y)\left(1-\gamma\left(\bar{\gamma}^{-1}(y)\right)\right)} \mathrm{d} y\right\} \exp \left\{-\Phi(q)\left(\bar{\gamma}^{-1}(a)-x\right)\right\} \\
&=\exp \left\{-\int_{x}^{a} \frac{W^{(q) \prime}(y)}{W^{(q)}(y)\left(1-\gamma\left(\bar{\gamma}^{-1}(y)\right)\right)} \mathrm{d} y\right\},
\end{aligned}
$$

where we have also used the fact that

$$
\int_{x}^{a} \frac{1}{1-\gamma\left(\bar{\gamma}^{-1}(y)\right)} \mathrm{d} y=\bar{\gamma}^{-1}(a)-\bar{\gamma}^{-1}(x)=\bar{\gamma}^{-1}(a)-x .
$$

The proof is now complete. 
Proof of Theorem 1.2. The proof builds on the experience of the calculations in the previous proof. We note that the process $S$ does not increase on the time interval $\left(L_{L_{\tau_{0}^{-}}^{-1}}^{-1}, \tau_{0}^{-}\right)$and, hence,

$$
\begin{aligned}
\mathrm{E}_{x}\left[\int_{0}^{\tau_{0}^{-}} \mathrm{e}^{-q u} \gamma\left(S_{u}\right) \mathrm{d} S_{u}\right] \\
=\mathrm{E}_{x}\left[\int_{0}^{L_{L_{\tau}^{-}}^{-1}} \mathrm{e}^{-q u} \gamma\left(L_{u}+x\right) \mathrm{d} L_{u}\right] \\
=\mathrm{E}_{x}\left[\int_{0}^{\infty} \mathbf{1}_{\left\{t<L_{\tau_{0}^{-}}\right.} \mathrm{e}^{-q L_{t}^{-1}} \gamma(t+x) \mathrm{d} t\right] \\
=\int_{0}^{\infty} \mathrm{E}_{x}\left[\mathrm{e}^{-q L_{t}^{-1}} \mathbf{1}_{\left.\left\{\bar{\varepsilon}_{s} \leq \bar{\gamma}(x+s) \text { for all } 0 \leq s \leq t\right\}\right]} \gamma(t+x) \mathrm{d} t\right. \\
=\int_{0}^{\infty} \mathrm{e}^{-\Phi(q) t} \mathrm{P}_{x}^{\Phi(q)}\left(\bar{\varepsilon}_{s} \leq \bar{\gamma}(x+s) \text { for all } 0 \leq s \leq t\right) \gamma(t+x) \mathrm{d} t \\
=\int_{0}^{\infty} \mathrm{e}^{-\Phi(q) t} \exp \left\{-\int_{0}^{t} n_{\Phi(q)}(\bar{\varepsilon}>\bar{\gamma}(x+s)) \mathrm{d} s\right\} \gamma(t+x) \mathrm{d} t \\
=\int_{0}^{\infty} \mathrm{e}^{-\Phi(q) t} \exp \left\{-\int_{0}^{t} \frac{W_{\Phi(q)}^{\prime}(\bar{\gamma}(x+s))}{W_{\Phi(q)}(\bar{\gamma}(x+s))} \mathrm{d} s\right\} \gamma(t+x) \mathrm{d} t \\
=\int_{0}^{\infty} \exp \left\{-\int_{0}^{t} \frac{W^{(q) \prime}(\bar{\gamma}(x+s))}{W^{(q)}(\bar{\gamma}(x+s))} \mathrm{d}\right\} \gamma(t+x) \mathrm{d} t
\end{aligned}
$$

where in the fifth equality the measure $n_{\Phi(q)}$ plays the role of $n$ under $\mathrm{P}^{\Phi(q)}$, in the penultimate equality we have used (2.4), and the final equality uses (2.7). The proof is completed by applying a straightforward change of variables.

Before turning to the proof of Theorem 1.3, we need first to prove an additional auxiliary result. To this end, define $\rho_{a}=\inf \{s>0: \varepsilon(s)>a\}$, the first passage time above $a$ of the canonical excursion $\varepsilon$. We also need the first passage times for the underlying Lévy process $X$,

$$
T_{x}^{+}=\inf \left\{t>0: X_{t}>x\right\} \quad \text { and } \quad T_{x}^{-}=\inf \left\{t>0: X_{t}<x\right\} \quad \text { for all } x \in \mathbb{R} \text {. }
$$

Lemma 2.2. For any $z>0, y \in[0, a]$, and $q \geq 0$, we have

$$
\begin{aligned}
& n\left(\mathrm{e}^{-q \rho_{a}} ; a-\varepsilon\left(\rho_{a}-\right) \in \mathrm{d} y, \varepsilon\left(\rho_{a}\right)-a \in \mathrm{d} z\right) \\
& =\left\{W^{(q) \prime}(a-y)-\frac{W^{(q) \prime}(a)}{W^{(q)}(a)} W^{(q)}(a-y)\right\} v(y+\mathrm{d} z) \mathbf{1}_{\{y<a\}} \mathrm{d} y \\
& \quad+W^{(q)}(0+) v(a+\mathrm{d} z) \delta_{a}(\mathrm{~d} y),
\end{aligned}
$$

where $\delta_{a}(\mathrm{~d} y)$ is the Dirac measure assigning unit mass to the point a and

$$
n\left(\mathrm{e}^{-q \rho_{a}} ; \varepsilon\left(\rho_{a}\right)=a\right)=\frac{\sigma^{2}}{2}\left\{\frac{W^{(q) \prime}(a)^{2}}{W^{(q)}(a)}-W^{(q) \prime \prime}(a)\right\} .
$$

Proof. Recall that $Y=S-X$. For the latter process, introduce its first passage time

$$
\varsigma_{a}=\inf \left\{t>0: Y_{t}>a\right\} .
$$


By a classical application of the compensation formula (see, for example, the treatment of a related problem in [4]) we have, for $z>0, y \in[0, a)$, and $q \geq 0$,

$$
\begin{aligned}
& \mathrm{E}\left(\mathrm{e}^{-q \zeta a} ; a-Y_{S_{a}-} \in \mathrm{d} y, Y_{S_{a}}-a \in \mathrm{d} z\right) \\
& \quad=\mathrm{E}\left[\sum_{t \geq 0} \exp \left\{-q\left(L_{t-}^{-1}+\rho_{a}\left(\varepsilon_{t}\right)\right)\right\} \mathbf{1}_{\left\{\sup _{s<t} \bar{\varepsilon}_{s} \leq a, \rho_{a}\left(\varepsilon_{t}\right)<\sigma\left(\varepsilon_{t}\right), a-\varepsilon_{t}\left(\rho_{a}-\right) \in \mathrm{d} y, \varepsilon_{t}\left(\rho_{a}\right)-a \in \mathrm{d} z\right\}}\right] \\
& =\int_{0}^{\infty} \mathrm{E}\left[\mathrm{e}^{-q L_{t}^{-1}} \mathbf{1}_{\left\{\sup _{s \leq t} \bar{\varepsilon}_{s} \leq a\right\}}\right] \mathrm{d} t \cdot n\left(\mathrm{e}^{-q \rho_{a}} ; a-\varepsilon\left(\rho_{a}-\right) \in \mathrm{d} y, \varepsilon\left(\rho_{a}\right)-a \in \mathrm{d} z\right) \\
& =\int_{0}^{\infty} \mathrm{e}^{-\Phi(q) t} \mathrm{e}^{-n_{\Phi(q)}(\bar{\varepsilon}>a) t} \mathrm{~d} t \cdot n\left(\mathrm{e}^{-q \rho_{a}} ; a-\varepsilon\left(\rho_{a}-\right) \in \mathrm{d} y, \varepsilon\left(\rho_{a}\right)-a \in \mathrm{d} z\right) \\
& =\int_{0}^{\infty} \mathrm{e}^{-\Phi(q) t} \exp \left\{-\frac{W_{\Phi(q)}^{\prime}(a)}{W_{\Phi(q)}(a)} t\right\} \mathrm{d} t \cdot n\left(\mathrm{e}^{-q \rho_{a}} ; a-\varepsilon\left(\rho_{a}-\right) \in \mathrm{d} y, \varepsilon\left(\rho_{a}\right)-a \in \mathrm{d} z\right) \\
& =\int_{0}^{\infty} \exp \left\{-\frac{W^{(q) \prime}(a)}{W^{(q)}(a)} t\right\} \mathrm{d} t \cdot n\left(\mathrm{e}^{-q \rho_{a}} ; a-\varepsilon\left(\rho_{a}-\right) \in \mathrm{d} y, \varepsilon\left(\rho_{a}\right)-a \in \mathrm{d} z\right),
\end{aligned}
$$

where in the first equality the time index runs over local times and the sum is the usual shorthand for integration with respect to the Poisson counting measure of excursions, and, for the second equality, we needed the quasi-left continuity for subordinator $L^{-1}$.

On the other hand, according to Theorem 1 of [19], we have (recalling that $y \in[0, a)$ )

$$
\begin{aligned}
& \mathrm{E}\left(\mathrm{e}^{-q \zeta_{a}} ; a-Y_{S_{a}-} \in \mathrm{d} y, Y_{\varsigma_{a}}-a \in \mathrm{d} z\right) \\
& =\int_{0}^{\infty} \exp \left\{-\frac{W^{(q)^{\prime}}(a)}{W^{(q)}(a)} t\right\} \mathrm{d} t \\
& \quad \times\left(W^{(q)^{\prime}}(a-y)-\frac{W^{(q)^{\prime}}(a)}{W^{(q)}(a)} W^{(q)}(a-y)\right) v(y+\mathrm{d} z) \mathrm{d} y .
\end{aligned}
$$

By comparing the left- and right-hand sides of (2.8) and (2.9) we thus have

$$
\begin{aligned}
& n\left(\mathrm{e}^{-q \rho_{a}} ; a-\varepsilon\left(\rho_{a}-\right) \in \mathrm{d} y, \varepsilon\left(\rho_{a}\right)-a \in \mathrm{d} z\right) \\
& \quad=\left(W^{(q)^{\prime}}(a-y)-\frac{W^{(q) \prime}(a)}{W^{(q)}(a)} W^{(q)}(a-y)\right) v(y+\mathrm{d} z) \mathrm{d} y,
\end{aligned}
$$

as claimed.

In order to write down an identity for $n\left(\mathrm{e}^{-q \rho_{a}} ; a-\varepsilon\left(\rho_{a}-\right) \in \mathrm{d} y, \varepsilon\left(\rho_{a}\right)-a \in \mathrm{d} z\right)$ in the case that $y=a$ and $z>0$, note that this can only occur when the excursion immediately jumps from 0 at time 0 over the level $a$. This is not possible when $X$ has paths of unbounded variation; see, for example, [16]. However, when $X$ has paths of bounded variation, it is known (cf. [18] for example) that excursions begin with a jump and the entrance law is given by $d^{-1} v(\mathrm{~d} x)$. Here $d>0$ is the drift term of $X$ when it is uniquely written in the form $X_{t}=d t-\xi_{t}$, where $\xi$ is a pure-jump subordinator. It is also known (cf. Lemma 8.6 of [12]) that, when $X$ has paths of bounded variation, we may write $d^{-1}=W^{(q)}(0+)$ for all $q \geq 0$. Moreover, when $X$ has paths of unbounded variation, we have $W^{(q)}(0+)=0$ for all $q \geq 0$. It follows that we may now generically write, for $z>0$,

$$
\begin{aligned}
n\left(\mathrm{e}^{-q \rho_{a}} ; a-\varepsilon\left(\rho_{a}-\right) \in\{a\}, \varepsilon\left(\rho_{a}\right)-a \in \mathrm{d} z\right) & =n(\varepsilon(0+)-a \in \mathrm{d} z) \\
& =W^{(q)}(0+) v(a+\mathrm{d} z) .
\end{aligned}
$$


For the proof of the second part, we should first note that it is known (cf. [12, p. 212]) that the process $X$ creeps downwards if and only if $\sigma \neq 0$. It thus follows that $Y$ creeps upwards if and only if $\sigma \neq 0$. Henceforth, assume that $\sigma \neq 0$. The proof then follows the same reasoning, except in (2.8) we replace the event $\left\{a-Y_{\varsigma_{a}-} \in \mathrm{d} y, Y_{\zeta a}-a \in \mathrm{d} z\right\}$ by $\left\{Y_{\varsigma_{a}}=a\right\}$ on the left-hand side and $\left\{a-\varepsilon\left(\rho_{a}-\right) \in \mathrm{d} y, \varepsilon\left(\rho_{a}\right)-a \in \mathrm{d} z\right\}$ by $\left\{\varepsilon\left(\rho_{a}\right)=a\right\}$ on the right-hand side. Furthermore, as a replacement for (2.9) in the argument, we use instead

$$
\mathrm{E}\left(\mathrm{e}^{-q 5 a} ; Y_{5 a}=a\right)=\frac{\sigma^{2}}{2}\left\{\frac{W^{(q) \prime}(a)^{2}}{W^{(q)}(a)}-W^{(q) \prime \prime}(a)\right\} \int_{0}^{\infty} \exp \left\{-\frac{W^{(q) \prime}(a)}{W^{(q)}(a)} t\right\} \mathrm{d} t,
$$

which is taken from Theorem 2 of [19].

Proof of Theorem 1.3. We give the proof for the first identity. The proof of the second identity follows along exactly the same lines using the second part of Lemma 2.2 instead, and is left as an exercise for the reader.

In a similar spirit to the proof of Lemma 2.2 we may write, for a given open interval $B \subset(0, \infty)$,

$$
\begin{aligned}
& \mathrm{E}\left(\mathrm{e}^{-\alpha \kappa-\beta\left(\tau_{0}^{-}-\kappa\right)} ; S_{\tau_{0}^{-}}^{U} \in B, U_{\tau_{0}^{-}-} \in \mathrm{d} y,-U_{\tau_{0}^{-}} \in \mathrm{d} z\right) \\
& \quad=\mathrm{E}_{x}\left[\sum_{t \geq 0} \mathbf{1}_{\left\{L_{t-}^{-1}<\tau_{0}^{-}\right\}} \mathrm{e}^{-\alpha L_{t-}^{-1}} \mathbf{1}_{\left\{S_{L_{t-}^{-1}}^{U} \in B\right\}} \mathrm{e}^{-\beta\left(\tau_{0}^{-}-L_{t-}^{-1}\right)} \mathbf{1}_{\left\{S_{\tau_{0}^{-}}^{U}=S_{L_{t-}^{-1}}^{U}\right\}} \mathbf{1}_{\left\{U_{\tau_{0}^{-}-} \in \mathrm{d} y,-U_{\tau_{0}^{-}} \in \mathrm{d} z\right\}}\right] .
\end{aligned}
$$

Note, however, that on account of the fact that

$$
S_{L_{t-}^{-1}}^{U}=S_{L_{t}^{-1}}^{U}=x+t-\int_{x}^{L_{t}^{-1}} \gamma\left(S_{u}\right) \mathrm{d} S_{u}=x+t-\int_{x}^{x+t} \gamma(y) \mathrm{d} y=\bar{\gamma}(x+t)
$$

we have $\left\{S_{L_{t}^{-1}}^{U} \in B\right\}=\{\bar{\gamma}(x+t) \in B\}$. Note also that $L_{t}^{-1}=\tau_{\bar{\gamma}(x+t)}^{+}$and $L^{-1}$ is quasi-left continuous. Hence, applying the compensation formula we have

$$
\begin{aligned}
& \mathrm{E}\left(\mathrm{e}^{-\alpha \kappa-\beta\left(\tau_{0}^{-}-\kappa\right)} ; S_{\tau_{0}^{-}}^{U} \in B, U_{\tau_{0}^{-}-} \in \mathrm{d} y,-U_{\tau_{0}^{-}} \in \mathrm{d} z\right) \\
& =\mathrm{E}_{x}\left[\int_{0}^{\infty} \mathrm{d} t \cdot \mathrm{e}^{-\alpha \tau_{\bar{\gamma}(x+t)}^{+}} \mathbf{1}_{\{\bar{\gamma}(x+t) \in B\}} \mathbf{1}_{\left\{\tau_{\bar{\gamma}(x+t)}^{+}<\tau_{0}^{-}\right\}}\right. \\
& \left.\times n\left(\mathrm{e}^{-\beta \rho_{\bar{\gamma}(x+t)}} ; \bar{\gamma}(x+t)-\varepsilon\left(\rho_{\bar{\gamma}(x+t)}-\right) \in \mathrm{d} y, \varepsilon\left(\rho_{\bar{\gamma}(x+t)}\right)-\bar{\gamma}(x+t) \in \mathrm{d} z\right)\right] \\
& =\int_{B} \frac{\mathrm{d} \theta}{1-\gamma\left(\bar{\gamma}^{-1}(\theta)\right)} \mathrm{E}_{x}\left[\mathrm{e}^{-\alpha \tau_{\theta}^{+}} \mathbf{1}_{\left\{\tau_{\theta}^{+}<\tau_{0}^{-}\right\}}\right] n\left(\mathrm{e}^{-\beta \rho_{\theta}} ; \theta-\varepsilon\left(\rho_{\theta}-\right) \in \mathrm{d} y, \varepsilon\left(\rho_{\theta}\right)-\theta \in \mathrm{d} z\right),
\end{aligned}
$$

where in the final equality we have applied a change of variable. Now making use of the first part of Lemma 2.2 and the conclusion of Theorem 1.1 the result follows.

\section{Acknowledgements}

The first author acknowledges the support of EPSRC grant number EP/D045460/1. The second author is supported by an NSERC grant. Both authors would like to thank an anonymous referee and J. F. Renaud for their comments on an earlier version of this paper. 


\section{References}

[1] Albrecher, H. ANd Hipp. C. (2007). Lundberg's risk process with tax. Blätter der DGVFM 28, 13-28.

[2] Albrecher, H., Renaud, J.-F. and Zhou, X. (2008). A Lévy insurance risk process with tax. J. Appl. Prob. 45, 363-375.

[3] Albrecher, H., Borst, S., Boxma, O. and Resing, J. (2009). The tax identity in risk theory-a simple proof and an extension. Insurance Math. Econom. 44, 304-306.

[4] Avram, F., Kyprianou, A. E. And Pistorius, M. R. (2004). Exit problems for spectrally negative Lévy processes and applications to (Canadized) Russian options. Ann. Appl. Prob. 14, 215-238.

[5] Avram, F., Palmowski, Z. and Pistorius, M. R. (2007). On the optimal dividend problem for a spectrally negative Lévy process. Ann. Appl. Prob. 17, 156-180.

[6] Bertoin, J. (1996). Lévy Processes. Cambridge University Press.

[7] Chan, T., Kyprianou, A. E. And Savov, M. (2009). Smoothness properties of scale functions for spectrally negative Lévy processes. Preprint.

[8] Chaumont, L., Kyprianou, A. E. and Pardo, J. C. (2009). Some explicit identities associated with positive self-similar Markov processes. Stoch. Process. Appl. 119, 980-1000.

[9] Hubalek, F. And Kyprianou, A. E. (2008). Old and new examples of scale functions for spectrally negative Lévy processes. Preprint. Available at http://arxiv.org/abs/0801.0393.

[10] HuZak, M., Perman, M., ŠIKić, H. ANd VondračeK, Z. (2004). Ruin probabilities and decompositions for general perturbed risk processes. Ann. Appl. Prob. 14, 1378-1397.

[11] Klüppelberg, C., Kyprianou, A. E. And Maller, R. A. (2004). Ruin probabilities and overshoots for general Lévy insurance risk processes. Ann. Appl. Prob. 14, 1766-1801.

[12] Kyprianou, A. E. (2006). Introductory Lectures on Fluctuations of Lévy Processes with Applications. Springer, Berlin.

[13] Kyprianou, A. E. And Loeffen, R. (2009). Refracted Lévy processes. To appear in Ann. Inst. H. Poincaré Prob. Statist.

[14] Kyprianou, A. E. and Palmowski, Z. (2007). Distributional study of de Finetti's dividend problem for a general Lévy insurance risk process. J. Appl. Prob. 44, 428-443.

[15] Kyprianou, A. E. And Rivero, V. (2008). Special, conjugate and complete scale functions for spectrally negative Lévy processes. Electron. J. Prob. 13, 1672-1701.

[16] Millar, P. W. (1977). Zero-one laws and the minimum of a Markov process. Trans. Amer. Math. Soc. 226, 365-391.

[17] Patie, P. (2009). Exponential functional of a new family of Lévy processes and self-similar continuous state branching processes with immigration. Bull. Sci. Math. 133, 355-382.

[18] Pistorius, M. (2004). On exit and ergodicity of the spectrally one-sided Lévy process reflected at its infimum. J. Theoret. Prob. 17, 183-220.

[19] Pistorius, M. R. (2007). An excursion-theoretical approach to some boundary crossing problems and the Skorokhod embedding for reflected Lévy processes. In Séminaire de Probabilités XL (Lecture Notes Math. 1899), Springer, Berlin, pp. 287-307.

[20] Renaud, J.-F. (2009). The distribution of tax payments in a Lévy insurance risk model with a surplus-dependent taxation structure. Insurance Math. Econom. 45, 242-246.

[21] Renaud, J.-F. And Zhou, X. (2007). Distribution of the present value of dividend payments in a Lévy risk model. J. Appl. Prob. 44, 420-427.

[22] Surya, B. A. (2008). Evaluating scale functions of spectrally negative Lévy processes. J. Appl. Prob. 45, 135149. 